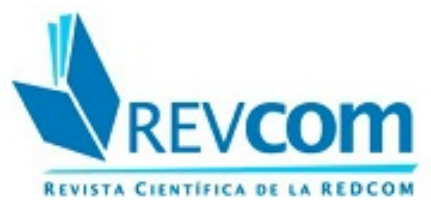

REVCOM. Revista científica de la red de carreras de Comunicación Social

ISSN: 2451-7836

redcom.revcom@gmail.com

Universidad Nacional de La Plata

Argentina

\title{
Una mirada desde la democracia deliberativa sobre medios, tecnologías y ensayos regulatorios en Uruguay
}

Alonso, Eduardo; Beltramelli, Federico

Una mirada desde la democracia deliberativa sobre medios, tecnologías y ensayos regulatorios en Uruguay

REVCOM. Revista científica de la red de carreras de Comunicación Social, núm. 10, 2020

Universidad Nacional de La Plata, Argentina

DOI: https://doi.org/10.24215/24517836e027 


\section{Una mirada desde la democracia deliberativa sobre medios, tecnologías y ensayos regulatorios en Uruguay}

Um olhar da Democracia deliberativa sobre meios, tecnologias e ensaios regulatórios no Uruguai

A look from the deliberative Democracy on media, technologies and regulatory trials in Uruguay

Eduardo Alonso

DOI: https://doi.org/10.24215/24517836e027

Facultad de Información y Comunicación Universidad de la

República, Uruguay, Uruguay

eduardo.alonso@fic.edu.uy

Recepción: 04 Mayo 2020

Federico Beltramelli

Aprobación: 24 Mayo 2020

Facultad de Información y Comunicación Universidad de la

República, Uruguay, Uruguay

federico.beltramelli@fic.edu.uy

Recepción: 04 Mayo 2020

Aprobación: 24 Mayo 2020

\section{Resumen:}

La Democracia deliberativa aborda, entre sus principales desafíos, a las limitaciones comunicacionales contemporáneas que genera la emergencia de las TIC. El escenario que impone la pandemia en curso modifica los comportamientos sociales y a los supuestos democráticos que la sostienen. Desde el estudio de caso del Uruguay se pretende analizar dichos impactos en la calidad democrática, su sistema comunicacional social y sus proyecciones de corto y mediano alcance.

Palabras Clave: pandemia, democracia deliberativa, comunicación social en Uruguay.

\section{Abstract:}

Deliberative Democracy refers among its main challenges to the contemporary communicational limitations generated by the emergence of ICT. The scenario imposed by the ongoing pandemic modifies social behaviors and the democratic assumptions that sustain it. The purpose of the Uruguay case study is to analyze these impacts on democratic quality, its social communication system and its close and mediate projections.

KEYWORDS: Pandemic, deliberative democracy, social communication in Uruguay.

\section{Resumo:}

A democracia deliberativa aborda, entre seus principais desafios, às limitações comunicacionais contemporâneas geradas pelo surgimento das TIC. O cenário imposto pela pandemia em curso modifica os comportamentos sociais e os pressupostos democráticos que a sustentam. O estudo de caso do Uruguai visa analisar esses impactos na qualidade democrática, em seu sistema de comunicação social e em suas projeções de curto e médio prazo.

Palavras-chave: Pandemia, democracia deliberativa, comunicação social no Uruguai.

\section{INTRODUCCIÓN}

El íntimo vínculo entre democracia y medios de comunicación cuenta con una extensa literatura en la comunidad académica y hoy día no es posible disociar la idea de la dependencia y causalidad que la primera tiene con estos últimos. De hecho, en las definiciones de la Poliarquía de Robert Dahl, además de las garantías procedimentales exigidas por la teoría democrática, incorpora la presencia indispensable del ejercicio del derecho a informarse, de comunicar y de expresarse. Pero ambas categorías vienen sufriendo una disminución en la apreciación pública. 
Previo a la irrupción de la pandemia del Coronovirus (COVID-19) que monopoliza a la atención mundial, amplios sectores de la literatura especializada en Teoría Política se encontraban preocupados por las debilidades crecientes de las democracias representativas y su pérdida de legitimidad. En respuesta, desde fines del siglo XX se han venido imponiendo diseños institucionales basados en los principios de una democracia deliberativa ${ }^{1}$. Estas alternativas han sido valoradas como mecanismos de refortalecimiento democrático a través de debates públicos intersubjetivos, dialógicos y plurales. Todas las propuestas, aunque con matices, requieren de sistemas de medios de comunicación masivos eficientes y fundamentalmente plurales que den cuenta de las razones involucradas en la toma de decisiones. A la tradicional dependencia de la democracia respecto a la necesidad de comunicar e informar, estos modelos precisan aún un mayor compromiso de los medios para difundir y promover deliberaciones sobre asuntos públicos de forma de fortalecer una política basada en razones y no solo en agregación de preferencias, agonística o centrada en intereses. Cabe aquí también referir a la necesidad de contar con sistemas regulatorios eficientes en la promoción de esos derechos y una institucionalidad fiscalizadora bajo estándares apropiados y con capacidades instaladas.

La irrupción de la pandemia, impredecible para los cientistas sociales, ha afectado el comportamiento social y algunos supuestos del debate democrático se han visto afectados por la modificación de conductas en la sociedad, atemorizada por un enemigo invisible y con proyecciones aún inciertas. Cabe preguntarse, ¿por qué razón la emergencia sanitaria afectaría el debate teórico en la materia? ¿Por qué un fenómeno tan contingente influiría en el análisis de comportamientos políticos, sociales y antropológicos en el largo plazo? Podemos ensayar una primera aproximación común a ambas preguntas. Porque la pandemia obligó a un cambio radical en las formas de convivencia de las sociedades y por lo tanto modificó el peso específico de las instituciones democráticas, por lo menos en el momento de desarrollo del fenómeno. Dentro de esos cambios relacionales y más allá de la casuística internacional, se destaca la capacidad mediática desplegada y la concentración de recursos de poder en los gobiernos, quienes asumen una mayor centralidad en el nuevo escenario. De forma que la pandemia crea condiciones de empoderamiento de los gobiernos de turno y exacerba una demanda compulsiva por información de fuentes confiables, que identifica fundamentalmente en los medios tradicionales de la comunicación, volviendo hacia una centralidad cuestionada por algunas tecnologías de la información y comunicación. Este fenómeno, revierte un proceso por el cual las redes sociales venían posicionándose como principal fuente de información del ciudadano. Si bien, aún no existen mediciones que den cuenta de estos fenómenos en términos absolutos es posible plantear algunas hipótesis sobre estos supuestos.

En el caso uruguayo, estos efectos recayeron en un novel gobierno recientemente electo (centro-derecha), de signo diferente a su antecesor (centro-izquierda), quien había asumido el Poder Ejecutivo apenas un par de semanas antes que llegara el primer caso al territorio. Toda la institucionalidad tradicionalmente reconocida por los analistas y comparativistas se vio repentinamente tensada. Cuando se esperaban los debates sobre la regulación de los medios, la rotación de autoridades de diferentes partidos que ostentaban una retórica radicalizada y las reacciones de la opinión pública desacostumbrada a tanta variación, se desarrolla una escenografía donde todos los parámetros de relacionamientos políticos y sociales se ven abruptamente modificados.

En el presente artículo, intentaremos retomar la línea reflexiva que involucra a la viabilidad de la democracia, tomando como variables independientes sus desafíos actuales, la proyección de escenarios potenciales de las sociedades que se reorganizan en una "nueva normalidad" 2 , poniendo el foco en las modalidades de comunicación de los asuntos públicos o comunes. Como referencia ejemplar de estas reflexiones, tomaremos a Uruguay como caso, dado su prestigio en materia de cultura política y por la doble contingencia de renovación de autoridades y emergencia sanitaria que coloca a los desafíos en máxima tensión. 


\section{1.- LOS DEBATES DEMOCRÁticos EN LA LITERATURA ESPECIALIZADA PREVIOS A LA CRISIS SANITARIA}

La democracia representativa o liberal viene reflejando en los diferentes indicadores comparados regionales e internacionales un evidente debilitamiento en la legitimidad ciudadana (Latinobarómetro, LAPOP, Eurobarometer, BTI Bertelsmann Stiftung Transformation Index, Banco Mundial, Freedom House, etc.) ${ }^{3}$. También todas las instituciones asociadas a la democracia como los parlamentos, los gobiernos, los partidos políticos, las organizaciones sociales de masas como cámaras empresariales y sindicatos laborales.

A la luz de estos indicadores y otros de carácter cualitativos y cuantitativos en el mismo sentido, en el ocaso del siglo XX comenzaron a difundirse investigaciones que interpelan los supuestos normativos y las reglas procedimentales que caracterizan a las democracias contemporáneas y proponen una modificación más o menos sustantiva de la organización de éstas.

Entre ellas se encuentra la denominada Democracia Deliberativa, que sostiene en algunos de sus postulados más aceptados, que la recuperación de la legitimidad ciudadana solo es posible si la política retoma o comienza a recorrer por los estándares de la deliberación racional para orientar la acción pública. En versiones más radicales, esa deliberación es además, un atributo ciudadano y por lo tanto refieren a una deliberación amplia y pública. Las promesas normativas de la deliberación en el robustecimiento de una cultura racional y mejor relacionada con la sociedad fijan los estándares deliberativos que se han venido revisando y decodificando desde sus supuestos teóricos de los años noventa hacia propuestas de diseños institucionales empíricos, que habiliten amplios o más eficientes ambientes de una política basada en razones y argumentos, en detrimento de concepciones de democracia como agregación de intereses o agonísticas que se regulan por los principios de mercado.

Pero la toma de decisiones basadas en razones y deliberadas no se presentan químicamente puras o concentradas en un único ambiente deliberativo, sino que serían incentivadas a lo largo del sistema político y de la sociedad civil (democracia deliberativa sistémica, John Dryzek, 2009) en relaciones dinámicas de intercambios o subida y bajada de argumentos que aportan a los decisores y a los potenciales afectados suficientes razones para aceptar sus definiciones. E incluso, existen diseños que articulan experiencias deliberativas en formatos de mini públicos o encuestas deliberativas (deliberative polls) ${ }^{4}$. Otros, señalan que el rol mediático de la retórica política -propia del sistema- asume en determinados momentos el formato de una deliberación razonada y basada en argumentos dialógicos (Iris Marión Young. 2000:53; Hartmut Wessler, 2008:4; Simone Chambers, 2009). Jürgen Habermas, en cambio, sostiene que la deliberación se cultiva en la sociedad civil en sus diferentes ámbitos y que ellas actúan como sensores del sistema político liberal. Aquí no se presenta una deliberación participativa, sino que esos intercambios argumentativos se elevan a la esfera pública a través de la influencia en los tomadores de decisión aunque no participen en ella. Es para Habermas el mecanismo para la expansión de una cultura deliberativa.

Sin embargo, todas estas visiones democráticas deliberativas soslayan o subvaloran el rol de las mismas instituciones políticas como promotoras de una cultura política razonada basada en la deliberación pública. Será recién a finales de la segunda ola del giro deliberativo, en estos últimos años, que surgen investigaciones orientadas a demostrar que las instituciones políticas y particularmente la que dispone de mayores capacidades para la construcción razonada y deliberada de decisiones públicas son los partidos políticos, sin ignorar que en la actualidad los partidos mayoritariamente se encuentran con déficit de identidades y que sus élites están indisolublemente asociadas a la propia gestión del estado y menos sujetas a controles ciudadanos (Katz y Mair, 1995). Pero eso no implica que en el pasado se identifiquen períodos donde los partidos jugaron ese rol constructor de identidades para producir comunidad en sus militantes y simpatizantes en base a la deliberación colectiva. Ni tampoco que no los haya contemporáneamente en determinados partidos o movimientos o que se encuentren impedidos a volver a hacerlo en el futuro (Jan Teorell, 1999; Fabio 
Wolkenstein, 2016 ; María Haberer e Ismael Peña-López, 2016; Enrico Biale y Valeria Ottonelli, 2018; Eduardo Alonso, 2019; entre otros).

La deliberación pública, coinciden todas estas corrientes, debe cumplir algunas condiciones imprescindibles para generar el efecto positivo que prometen sus postulados normativos. Las instancias deliberativas deben ser dialógicas, plurales, democráticas y públicas o conocidas por todos los afectados de la decisión. De tal forma que cuando se habla de democracia deliberativa, se deben incorporar aquellos elementos comunicativos de los fundamentos deliberados que sostienen una decisión. Es decir, que no sería suficiente que las élites de un partido político tuvieran la voluntad política de someter el sistema de decisiones de la organización en ambientes abiertos y deliberativos, sino que también requiere que esos debates sean suficientemente difundidos públicamente. Este último aspecto, es posiblemente una de las mayores encrucijadas a la que se enfrentan los partidos políticos tradicionales contemporáneos o en las otras versiones democráticas deliberativas, la sociedad en su conjunto. El mensaje no debe ser cerrado, sino que debe incorporar los fundamentos y para ellos se requieren de medios de comunicación potentes.

Los medios de masas han sido históricamente interpelados por su capacidad de editar, filtrar o dirigir los mensajes, muchas veces sospechados por informar en función de sus propios intereses o en representación de intereses privados o corporativos, pero sin embargo, han sido capaces de concentrar las audiencias a lo largo del tiempo. Asumimos junto a Califano que «las relaciones entre política, medios y sociedad se encuentran atravesadas por la regulación del Estado en materia de políticas de comunicación» (Califano, 2015: 283). Esta tensión entre los aspectos normativos de la comunicación democrática y la presencia de poderosos intereses económicos, sociales o políticos, representan los tipos o calidades de sistemas mediáticos más o menos permeables a unas y otras funciones. Los incentivos estudiados en el pasado han ido en ambas direcciones. Los grupos editoriales oscilan entre sus intereses comerciales y sus funciones democráticas.

En todo caso, la irrupción de la internet y las aplicaciones asociadas a la comunicación e información han modificado la inexorabilidad de que la comunicación pública sea tamizada por los grandes medios de comunicación. Un beneficio inmediatamente reconocible de las redes sociales y los portales on line ha sido la diversificación de las fuentes y el control ya no estaría supeditado exclusivamente a los medios tradicionales. Sin embargo, esta virtud de internet se ha vuelto también una limitación en libertad de información de los ciudadanos, ante la vulnerabilidad frente a información sucia o de fuentes desconocidas o de dudosa procedencia y sus efectos tan prometedores se han visto moderados.

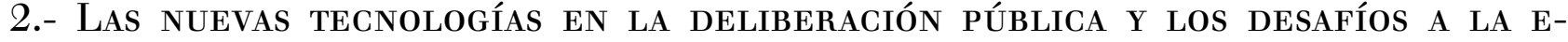 DEMOCRACIA}

Si la deliberación pública fortalece a las democracias y sus instituciones visibles, ¿cuál es el efecto de la expansión de internet y el uso de las redes sociales respecto a la deliberación pública?, y ¿qué residuo dejan los medios tradicionales en esta contingencia?

Giovanni Sartori lanzaba al final de su "Homovidens: la sociedad teledirigida" (1999) un desafío que hasta el momento aparecía extremadamente contraintuitivo, por la cual la televisión es una tecnología que modifica más el comportamiento antropológico que la internet. Se presenta como una tecnología superior respecto a la formación de la opinión de las personas. La apuesta que esgrime Sartori, es que la 'realidad' que exhibe la televisión es aún fuertemente demandada y ello parece concordante con el clima de consumo actual, particularmente valorando a los ciudadanos en cuarentena. Las redes sociales parecen perder su potencial como fuente de información principal, a partir del agrietamiento del grado de legitimidad frente a la voz del sistema político y en particular de los gobernantes que enfrentan la pandemia a través de los medios de masas tradicionales como la televisión y la radio.

Pero el universo de consumo informativo tiene entusiastas de ambos lados del mostrador, es así que Joan Subirats (2015) en un mundo pre Covid-19, a contrapelo de lo esgrimido por Sartori, coloca en el centro 
de su análisis sobre la realidad política española el dilema deliberativo al interior de los partidos frente a los desafíos tecnológicos. En el caso español contemporáneo, los partidos de nuevo cuño (Podemos, Ciudadanos, Vox) desarrollan una gran capacidad para generar espacios de deliberación horizontal a partir del uso de las nuevas tecnologías de las comunicaciones. El uso de las redes sociales virtuales que se reconocen dentro de la institucionalidad de los nuevos partidos, promueven una apertura de la política a ciudadanos proactivos y coincide con la entrada en crisis de los partidos tradicionales. Subirats sostiene que "Internet, no es sólo un espacio de nuevas tecnologias de la información y la comunicación. Es un espacio nuevo, que rompe con maneras de hacer y erosiona a muchas organizaciones e instituciones que no logran mantener el valor añadido de su intermediación en el escenario digital. En ese sentido, la idea de "nueva politica" convendría enmarcarla en ese escenario de cambio de época y, sobre todo, de cambio tecnológico".

Joan Subirats asume, entonces, la defensa de los recursos interactivos característicos de las redes virtuales como forma de crear y ampliar espacios públicos y fundamentalmente, tanto como que las nuevas tecnologías impactan directamente en la promoción de la deliberación al interior de los partidos políticos, y desafía a una literatura mayormente desconfiada de las promesas más audaces de los cultores digitales de los años 90. En la extensa y diversa propuesta académica del género se pueden reconocer algunas sugerencias taxonómicas con un alto grado de consenso de la literatura especializada. De hecho, Fernando Harto (2006) nos ofrece una buena síntesis de la taxonomía existente en materia de democracia digital o e-democracia. Si bien la variedad de criterios considerados en cada una de las categorizaciones, encuentra que existen en todas ellas un patrón que reduce el conjunto de aportes académicos en dos grandes visiones sobre la e-democracia: aquellos que suponen que la expansión tecnológica provocará la muerte definitiva de la democracia representativa tal cual la conocemos actualmente, y quienes sostienen que los desarrollos tecnológicos vienen a complementar y mejorar la versión de la democracia representativa. Entre los primeros, es común observar modelos de democracia directa con diferentes inspiraciones pero que suponen una modificación sustancial de la gestión pública y la naturaleza de los gobiernos, junto a la desaparición de la intermediación entre el ciudadano y la esfera pública. A los efectos de nuestra investigación, nos focalizaremos en los segundos.

Desde las primeras reflexiones que destacaban el vértigo y tendencia de las TIC permeadas de una euforia inicial sobre el alcance que se nos imponía en los aportes clásicos de Nicolás Negroponte (1995) y Manuel Castells (2000) hasta nuestros días, las investigaciones postreras han encontrado severas limitaciones de aquellas expectativas en lo que refiere al intercambio de razones en procesos de acumulación de argumentos que derivan en una decisión política (Andrew Chadwick, 2009). La accesibilidad de la comunicación en redes, adolecen de cierto diseño en burbujas debido a la reproducción endogámica de intereses. Para el interés de artículo podemos afirmar siguiendo a Sunstein (2001), que esas redes y grupos de discusión suelen intercambiar puntos de vista desde una misma perspectiva, constituyéndose en un factor excluyente de visiones diferentes inhibiendo una de las condiciones fundamentales del ideal deliberativo, como es la diversidad de puntos de vista.

El fenómeno también se reproduce en las fuentes de información que provee la Internet, porque el acceso fácil y libre al universo informativo genera que las personas intuitivamente buscan un tipo de información acorde a su sensibilidad y fuertemente vinculado a sus propios intereses filtrando aquello que no le interesa, cumpliéndose de esta forma la profecía de Nicolás Negroponte: la construcción del "The Daily me" (diario personalizado). Debemos agregar, que lo que el consumidor no busca, el algoritmo se lo prepara, sobremanera si consideramos que la economía informativa está sujeta a una intermediación por plataformas como Google, Facebook, Amazon, Uber (Srnicek, 2018), donde la sistematización, y capitalización de la información del usuario brinda a las corporaciones la posibilidad de adelantarse a posicionamientos y conductas de consumo de quienes acceden a las mismas. Esto último, puede implicar la predicción de escenarios de debates, coartando el libre juego en la configuración de la opinión pública. Desde el punto de vista de la expectativa deliberativa ese fenómeno tiene daños colaterales severos. El efecto se invierte y el acceso libre y la capacidad 
de filtrar se transforma en la renuncia a informarse sobre asuntos y temas que no lo convocan. Cass Sunstein propone dos soluciones para que se den las condiciones apropiadas a una deliberación democrática:

First, people should be exposed to materials that they would not have chosen in advance. Unplanned, unanticipated encounters are central to democracy itself. Second, many or most citizens should have a range of common experiences. Without shared experiences, a heterogeneous society will have a much more difficult time in addressing social problems. People may even find it hard to understand one another. Common experiences, emphatically including the common experiences made possible by the media, provide a form of social glue. (2001:10-11)

En la misma perspectiva, el trabajo de Lincoln James Dahlberg (2000) que busca parangonar las exigencias de las definiciones de la esfera pública habermasiana para la conformación de una cultura deliberativa, marca las limitaciones y las tendencias privatizadoras de los foros virtuales, así como un creciente control por los intereses económicos o corporativos. Pese a ello sostiene que el concepto de esfera pública habermasiano sigue siendo un buen marco de análisis de la Internet, existiendo espacios y foros que cumplen de forma bastante acabada con sus exigencias. Dahlberg destaca los efectos de las TICs como un complemento para las deliberaciones públicas tradicionales, como pueden ser los propios partidos políticos. Como Sunstein y Dahlberg, existe una corriente de trabajos que en el mismo sentido, identifican el uso de la internet más que como un espacio de expansión deliberativa, como un lugar en donde se profundizan los fenómenos de individualización, privatización y de mercado bajo una fuerte impronta liberal, pero reversibles a partir de la aplicación de una serie de propuestas en favor de una vigorización de los procesos democráticos y que surgen a partir de contrastar lo que denominan como Teledemocracia y democracia deliberativa (Ian Budge, 1993; Scott London, 1995; Enrique Cebrián Mazurca, 2005). Todos estos aportes se apoyan en diferentes investigaciones empíricas que identifican los usos en las redes.

El impacto cuantitativo y cualitativo del uso de las redes para incidir en la vida pública y en función de una comparación exhaustiva realizada por Jacob Nielsen (2006) estableció lo que se denomina Ley de potencia o la regla 90/9/1, por la que en los sitios WEB 2.0 el 90\% es lector pasivo, el $9 \%$ colabora muy poco y solo el $1 \%$ asume la responsabilidad del funcionamiento dinámico de la WEB. Esto ha sido interpretado en la literatura especializada como la verificación de la apatía o falta de compromiso del ciudadano contemporáneo con los temas públicos, pero también establece lo minoritario y selecto que resulta ser el ciudadano internauta proactivo.

Desde otra perspectiva, investigaciones orientadas hacia el marketing político destacan las opciones de Internet como herramientas de comunicación idóneas en las campañas de los partidos políticos y demuestran que efectivamente el uso de las TIC es generalizado, pero pocos de esos trabajos se detienen en el análisis de la funcionalidad de esos instrumentos para el uso de espacios para la deliberación. Este dato es congruente con la idea de que los comportamientos políticos no son modificados por la emergencia de nuevas tecnologías de comunicación, sino que estas adquieren relevancia cuando por fuera de ellas se piensa y valora el desarrollo de procedimientos deliberativos y resultan funcionales a esas convicciones.

\section{3.- LOS EFECTOS DE UNA PANDEMIA ANUNCIADA EN LA CALIDAD DEMOCRÁtica DE LAS SOCIEDADES}

La dinámica de una pandemia altamente letal y contagiosa produjo en nuestras sociedades evidentes impactos para no ser afectadas por ella o serlo en la menor forma posible. En particular, los procesos de cuarentena o aislación social que los gobiernos fueron imponiendo con diversos grados de rigurosidad y de celeridad. Estas medidas fueron convalidadas por las respectivas sociedades, generando una expansión del poder punitorio de los aparatos estatales, legitimadas por los fines superiores que supone la lucha contra la pandemia. De hecho, en sociedades con conflictos agudos se desarrollan escenarios donde los centros de poder se consolidan en la 
lucha contra la pandemia y han postergado los enfrentamientos con la oposición, beneficiando a quienes por el momento detentan el poder.

Frente a la novedad de la agresión de la pandemia los gobiernos se colocaron al frente de la batalla para el ojo del ciudadano, quién se somete acríticamente a los diagnósticos que estos les describen a través de los medios, así como consumen toda la información que aquellos proveen. En América Latina, este dato no sería una buena noticia desde el punto de vista democrático, ya que los gobiernos de regímenes presidenciales suelen concentrar en muy pocas personas el poder absoluto del estado. Por lo tanto, la centralidad de los gobiernos para decidir temas con altos contenidos científicos y con tanta afectación en el ciudadano de a pie por las decisiones que se asumen, constituyen la antítesis de la buena salud democrática, que requiere poderes controlados y mayores compromisos ciudadanos en la elaboración de las políticas.

En el mismo sentido, la exigencia de cuarentenas más o menos estrictas y el temor natural de las personas que observan el aluvión de información sobre la pandemia genera por lo menos dos efectos intuitivamente razonables. El primero es que los mecanismos de respuestas de las sociedades para instalar los resortes contramayoritarios se neutraliza y elimina o reduce la posibilidad de expresarse por los mecanismos tradicionales, que no implica una censura absoluta, pero sí una dispersión de los temas que la sociedad puede digerir por mecanismos alternativos a los de la protesta. En segundo lugar, muchos temas que eran de alta preocupación en la sociedad quedan relegados por la centralidad que asume la acción vinculada a la lucha contra la pandemia, que representa su principal fuente de preocupación. Esto permite la acción gubernamental sin mayores contralores en políticas que en condiciones normales hubieran requerido de negociaciones, campañas de difusión e implementación de políticas visibles para el conjunto de la sociedad.

Del mismo modo, la vida democrática que se nutre de la acción del conjunto de los partidos políticos del sistema ofrece una sobrerrepresentación mediática a los partidos en el gobierno y una subrepresentación de los partidos de oposición que opera en una ampliación mayor del actor gubernamental. Este fenómeno, es sin embargo, limitado en el tiempo porque las políticas que se exhiben exitosas al comienzo pueden sufrir también fracasos de la que serán muy expuestas a los ojos ciudadanos y una nueva oportunidad para las fuerzas de oposición quienes podrán encontrar nuevos espacios en los sistemas mediáticos tradicionales. El resultado es impredecible, pero el rol que juegan los medios adquiere una mayor centralidad que en tiempos menos amenazados.

Otro efecto, relacionado directamente con la calidad democrática es el que surge en forma pasiva al comienzo, que implica la parálisis del sistema económico en lo local, pero fundamentalmente, el fenómeno se desarrolla a nivel planetario. La relación entre los escenarios económicos adversos y los gobiernos de turno es muy íntima, como lo demuestra la literatura comparada. Por ello, es muy probable que el mundo observe una mayor movilidad de gobiernos y en algún caso, puede desencadenar en inestabilidad institucional. Los problemas económicos que se generan produce en la mayoría de los casos alta conflictividad social y los países con menor calidad institucional tendrán mayores desafíos para conservar las reglas del juego, mientras que aquellos sistemas políticos con mayor institucionalidad podrán procesar la crisis social que será inminente. Es muy difícil sostener que frente a la crisis global, un gobierno pueda renunciar a su responsabilidad y ello hace prever escenarios con importantes nubarrones en el horizonte.

Paralelamente a estos escenarios que se pueden describir en diferentes etapas, surgen las incertidumbres de cómo pueden jugar los medios en escenarios de conflicto. Como se afirmó inicialmente, en la primera etapa de lucha contra la pandemia, gobiernos y medios se ven favorecidos por la confianza de la opinión pública en información de fuentes confiables. Pero en períodos donde los centros de poder se vuelven inestables es importante conocer las fortalezas y las debilidades de los sistemas mediáticos, así como el rol que jugarán en el juego democrático.

Estas incertidumbres se acumulan frente al desarrollo de las tecnologías de la información y la comunicación. Se ha observado en el pasado reciente, que las redes sociales asumen cierto protagonismo como herramienta de comunicación social cuando los medios masivos tradicionales son percibidos como correa 
de transmisión del status quo. La primavera árabe, las grandes movilizaciones populares y relativamente espontáneas de los países occidentales de Europa como el 11M, el movimiento de los chalecos amarillos, movilizaciones en los Estados Unidos o más recientemente en Chile y en Hong Kong.

Curiosamente, si bien aún no existen suficientes datos empíricos sobre las formas de expresión de este comportamiento en tiempos de pandemia, se pueden adelantar algunas hipótesis sobre sus impactos. Una de las más llamativas, es que las redes sociales que se expanden por internet no respondieron eficientemente la demanda de información que estos nuevos ciudadanos de la "nueva normalidad" requieren. La falta de rigurosidad de mucha informaciones presentes en ellas, lo contradictorio de sus versiones, la evidencia de fake news y el aprovechamiento de la delincuencia cibernética minaron a las redes como fuentes principales de información. En cambio, los medios masivos de comunicación tradicionales retomaron la centralidad que la expansión de la internet les venía negando, asociados a la utilización que los gobiernos hicieron de ellos.

\section{4.- Medios masivos tradicionales Versus redes Sociales e Internet EN El URUguay: LOS ENSAYOS REGULATORIOS}

En Uruguay podemos constatar un sistema de medios estable, con un alto grado de concentración de grupos privados (Buquet y Lanza: 2011) que solo en televisión representa más del noventa por ciento de la audiencia y un sistema de medios públicos testigo. Las barreras entre los servicios de comunicación tradicionales y las tecnologías asociadas al dato e internet son altas y han impedido su trasvasamiento. Se puede afirmar que el sistema de medios refleja un 'periodismo de declaración' (Schuliaquer, 2016), abonado por una buena dosis de partidocentrismo en la agenda mediática. En el marco de la crisis del covid-19 se ha establecido una conferencia de prensa permanente por parte del Poder Ejecutivo, como también se amplió el formato de los informativos hasta un máximo de tres horas con altos niveles de audiencia. Debemos consignar que en Uruguay no existen canales especializados en noticias y la centralidad de los informativos nocturnos se refleja en ratings que superan a cualquier otra programación.

El intento de regulación de los servicios de comunicación audiovisual y los debates que le precedieron marcaron al período de gobierno dominado por el Frente Amplio en cuanto a medios de comunicación, con saldos de estabilidad en el sistema mediático, ya que la normativa mantuvo la división entre sectores y además su implementación fue deficiente y por momentos nula, lo que evitó afectar los intereses de los grupos mediáticos en las cláusulas que la ley sancionó para hacerlo. Mientras tanto, la empresa telefónica estatal ANTEL ${ }^{5}$ consolidaba sus infraestructuras en transmisión de datos en un ambiente de competencia controlada con la empresa Movistar (Telefónica) y Claro (Grupo Slim). En materia de fibra óptica, además, ANTEL contó con la complacencia del gobierno para usufructuar un monopolio que se fundamentaba en la extensión de la normativa que le dió su origen. De hecho el proceso de convergencia tecnológica en información y comunicación vino mayormente impulsado por los avances tecnológicos generados en el exterior, que por los intentos gubernamentales de regularlos.

Pero observemos en el largo plazo, como ha sido la relación entre medios y política en el Uruguay y sobre qué supuestos democráticos se fue articulando la matriz comunicacional y sus avances tecnológicos.

\section{Matriz comunicacional: cambios e inercias}

\section{Periodización:}

1) Matriz Comunicacional Republicana. 1900-1973

2) Matriz Comunicacional Transicional. 1980-1990

3) Matriz Comunicacional Contemporánea. 1991- 2020 
Características principales:

I) Matriz Comunicacional Republicana

a) Medios de comunicación y partidos eran el mismo actor. Cada fracción política de todos los partidos disponía de uno o más diarios, pero uno como mínimo.

b) Los dueños de los diarios eran los dirigentes políticos de la fracción o familiares o colaboradores íntimos al mismo.

c) Los contenidos reflejaban la vida social, cultural y fundamentalmente política de la sociedad. Esta última incorporaba: actas y debates parlamentarios, decisiones y debates del gobierno, funcionamiento y debate intrapartidario y voceros en los debates interpartidarios. Los adherentes de cada fracción se informaban de su partido y de los otros a través de dichos debates, cumpliendo con un exigente requisito fundamental de un sistema institucional republicano radical, que es sostenerse en una fuerte cultura cívica de los ciudadanos.

d) Con la aparición de la radio (desde la década del 30) se mantuvo la misma matriz, disponiendo cada fracción política de una o más radios para cumplir con los mismos fines que los gráficos, más allá que esa tecnología habilita a una gran oferta del espectro radioeléctrico con otros fines.

e) En los años 50 y 60 se distribuían más de 600.000 diarios cubriendo casi la totalidad del universo de hogares.

II) Matriz Comunicacional Transicional

a) Aparición y comienzo de la expansión de la Televisión Color

b) Despliegue en el mundo gráfico de los semanarios partidarios, sustituyendo a los diarios que ahora representaban "sensibilidades partidarias", pero no la voz de los partidos.

c) Desarrollo de las familias empresarias que abarcaban como grupo mediático a la Televisión, radios y diarios, con expresión de "sensibilidad partidaria", pero sin compromisos partidarios.

d) Mercantilización de los medios.

III) Matriz Comunicacional Contemporánea

a) Separación paulatina de los actores partidarios de los dueños de los medios. Partidos se debilitan, empresarios se fortalecen y los medios de comunicación se constituyen en actores privilegiados para la conformación de la agenda, por lo que cada gobierno debe negociar con los propietarios beneficios a cambio de un buen tratamiento en la misma.

b) Pérdida de sensibilidad política. La opinión pública se construye fundamentalmente por la discrecionalidad y los intereses económicos de los medios de comunicación

c) Los partidos se expresan con alta dependencia a través de los medios masivos y pierden o disminuyen sustantivamente su voz propia.

d) Consolidación de los grupos empresariales y constitución de un oligopolio de empresarios, fundamentalmente a través de la televisión abierta y con la irrupción de la ampliación de la oferta audiovisual de la televisión por abonados en la década del 90.

Estas matrices parecen acercarse a una nueva reformulación como efecto de la expansión de la Internet. Si bien es notorio que el proceso de convergencia de las tecnologías de información y comunicación concentra sus recursos en el marco de internet, este proceso está aún en desarrollo. Ello considerando dos aspectos fundamentales de la convergencia. El primero, es que aún no se percibe la magnitud de los impactos directos en la comunicación masiva, dado que las diferentes plataformas comunicacionales tradicionales continúan conviviendo con diferentes eficiencias en los nichos de la sociedad, como en determinadas franjas etarias, ubicación territorial de las personas o nivel socioeconómico, frente a la expansión comercial de los servicios que brinda la internet. El segundo, es que internet desarrolla sus aplicaciones en múltiples sentidos, aportando 
alternativas comunicacionales en cada uno de ellos mediante tecnologías de plataforma que funcionan como intermediarios a partir de efectos de red, con la consiguiente automatización y valorización de la información circulante (Srnicek, 2018). Pero aún así todavía no se vislumbra cómo se produce el acceso simultáneo a grandes espacios de la audiencia con las nuevas tecnologías. Si internet no logra llegar en forma homogénea a la opinión pública, los problemas comunes no podrán ser resueltos con los mismos insumos colectivamente, como se observaba en el capítulo anterior.

Desde el punto de vista de la calidad de la información ciudadana, estudios empíricos (Alonso, Beltramelli et all, 2018) dan cuenta que en los últimos diez años, la internet ha duplicado la preferencia de los uruguayos como principal fuente de información, aunque el uso mayoritario se concentra en los portales de los medios gráficos y televisivos tradicionales, en las franjas etarias más jóvenes en cambio, las redes sociales venían asumiendo su principal rol.

\section{¿Cuál considera que es su principal fuente de información?}

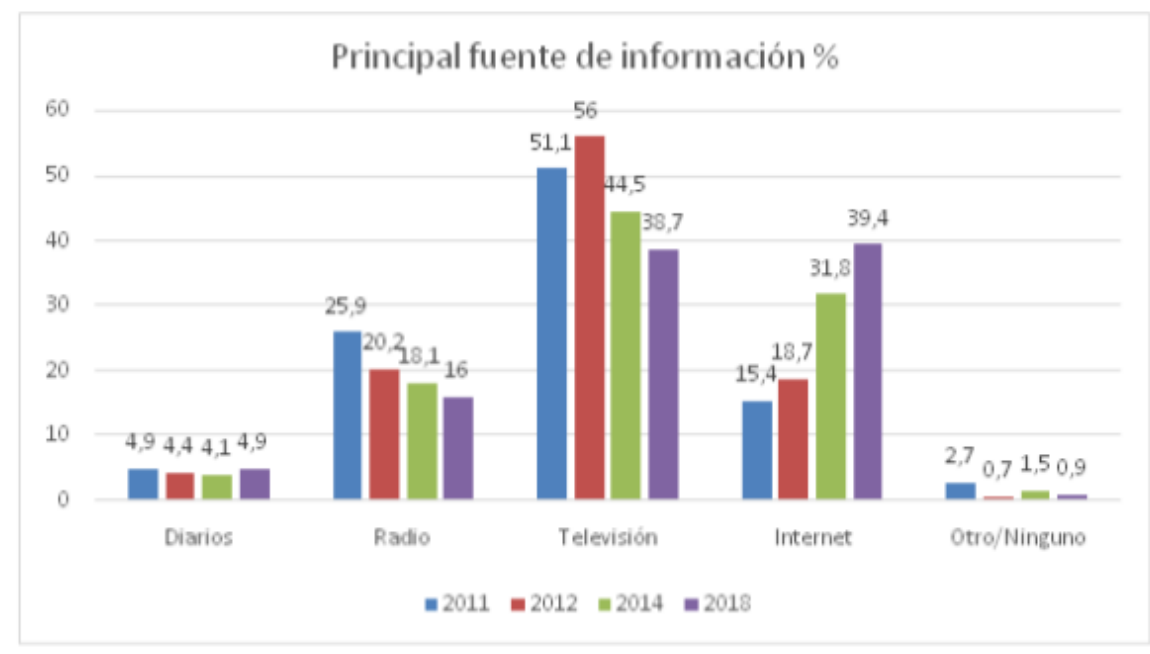

Fuente: Actualización al 2018 del informe "La ciudadanía ante la convergencia tecnológica y los medios de comunicación II. 2017” Beltramelli, Alonso, e Irigoin

Los comportamientos sociales vinculados a la comunicación no son tan homogéneos como lo expresa la gráfica. En el siguiente cuadro de contingencia, es posible percibir cómo los jóvenes tienen, como es de esperar, un vínculo mucho más fuerte con las redes de internet (dos de cada tres uruguayos) que los adultos y los adultos mayores. El informe publicado sostiene que

La edad de los consultados influye fuertemente en sus preferencias exhibiendo una relación estadísticamente significativa en forma inversa entre los extremos etarios. Diarios radios y particularmente la televisión son la fuente principal de información de la franja etaria de 60 años y más, mientras que la Internet es preferida por la amplia mayoría de los jóvenes de entre 18 y 29 años. En la franja de los adultos, la internet ya es más preferida que la tv.2 


\begin{tabular}{|l|l|l|l|l|l|l|l|}
\hline $\begin{array}{l}\text { Edad } \\
\text { agrupada }\end{array}$ & Diarios & Radio & Tv & Internet & Otro & Ninguno & Total \\
\hline 18 a 29 & $3,6 \%$ & $6,2 \%$ & $24,9 \%$ & $65,3 \%$ & $0,0 \%$ & $0,0 \%$ & $100,0 \%$ \\
\hline 30 a 59 & $4,6 \%$ & $15,0 \%$ & $33,7 \%$ & $45,1 \%$ & $0,8 \%$ & $0,8 \%$ & $100,0 \%$ \\
\hline 60 o más & $6,2 \%$ & $25,1 \%$ & $57,5 \%$ & $10,9 \%$ & $0,0 \%$ & $0,4 \%$ & $100,0 \%$ \\
\hline Total & $4,9 \%$ & $16,1 \%$ & $38,7 \%$ & $39,4 \%$ & $0,4 \%$ & $0,5 \%$ & $100,0 \%$ \\
\hline
\end{tabular}

CUADRO 2\#3 PRINCIPAL FUENTE DE INFORMACIÓN POR EDAD AGRUPADA

\section{El sistema de medios propuesto por la Coalición Multicolor liderada por el Partido Nacional}

En las recientes instancias electorales (2019), el cambio de gobierno presenta un escenario con un redireccionamiento de los vientos que dominaron el sistema de medios de comunicación y de competencia en las telecomunicaciones de los últimos 15 años. Estos cambios, exhiben algunas modificaciones precisas que se plantean en la modificación de la denominada ley de Servicios de Comunicación Audiovisual de diciembre del 2014 (ley $19307^{6}$ ), que fuera aprobada ya en el ocaso del gobierno del presidente José Mujica ${ }^{7}$.

Las reformas habilitarían a ampliar la competencia en el terreno de las telecomunicaciones en el que ahora podrán participar los permisarios de televisión por abonados con sus propias redes o arrendando las de la propia ANTEL, la implementación de la portabilidad numérica en la telefonía móvil asociada a un nuevo rol asignado a la empresa estatal ANTEL en la venta mayorista de transmisión de datos, bajo la obligación de disponibilizar la red de fibra instalada a cableoperadores ${ }^{8}$, lo que estaría indicando una apertura pro mercado, que llevaría a ANTEL como empresa pública estatal a perder el beneficio del monopolio en el servicios de internet por fibra.

Un análisis anticipado de cada una de estas reformas, da cuenta de un incentivo a la emergencia de nuevos actores en los diferentes modelos de negocios, amplitud en las reglas de competencia y un redireccionamiento del rol de la empresas del estado, así como el fortalecimiento mediante la asignación de una mayor autonomía técnica a la Unidad Reguladora de Servicios de Comunicaciones (URSEC), el organismo regulador de telecomunicaciones y medios audiovisuales.

\section{Cambios en la ley 19307 de Servicios de Comunicación Audiovisual del 2014.}

Uno de los aspectos más sobresalientes de esta reforma está en incluir en la ley la apertura para que los grupos que brindan servicios de televisión por abonados por cable, puedan utilizar sus redes para transmisión de datos. No es que existiera en el texto anterior un impedimento, sino que el mismo estaba en otras disposiciones que por vía de decretos o por resoluciones de la URSEC se había asentado el principio que ANTEL conservaría el monopolio de las redes de fibra óptica para transmisión de datos. De hecho, la reforma va más lejos, porque permite que ANTEL arriende a estas empresas su propia red de fibra óptica. El principio 
de sus redactores, es que la red de fibra óptica es un servicio público y como tal puede ser utilizado por cualquiera que pague un precio justo por el servicio prestado. ANTEL no deja de realizar el servicio de transmisión de datos, pero cumplirá un doble objetivo comercial: vender servicios de transmisión de datos en un mercado abierto a la competencia y a la vez, proveedor mayorista de otros usuarios que con fines comerciales quieran usar el servicio, incluso para competir con la propia ANTEL. En un principio no se trataría de una privatización, sino de colocar a la empresa estatal en un escenario de competencia en los servicios minoristas y también en los servicios mayoristas.

Otra de las modificaciones, si se quiere la más controversial desde el punto de vista mediático, es la que refiere al tope de concentración de medios por grupo empresarial. Hasta el momento, los grupos empresarios permisarios de la televisión abierta, tenían limitado su capacidad de expansión a no más de tres permisos por servicio, en la reforma se vuelve a subir esa limitación a ocho permisos (en la versión anterior a la ley 19.307 se autorizaba hasta seis). El punto no exhibe una buena calidad argumentativa por parte de sus redactores, salvo que le asiste la razón del artillero. La ley 19307 limitaba a los grupos existentes, pero nunca aplicó la norma y permitió en los hechos la continuidad con sus empresas y permisos que entraban dentro de la antigua legislación.

Más allá de las diferencias ideológicas que privilegian la acción del estado o la de mercado, existe un dilema que atraviesa a todos los gobiernos y que representan dos caras de una misma moneda. El mercado uruguayo es reducido y operaron barreras altas entre los sistemas de medios tradicionales y el mercado de datos e internet. Los arreglos políticos desarrollados hasta el momento traslucen un 'pacto criollo' de no agresión, por un lado el sistema de medios no se vio modificado o impactado por las ley de servicios audiovisuales de Frente Amplio, y por otro el mercado de internet por fibra se mantuvo bajo una lógica de monopolio natural de ANTEL. En esa línea, en los últimos gobiernos se pusieron límites y se accionó contra las multinacionales que dominan la televisión por abonados satelital más que limitar al oligopolio nacional, reflejada en la conocida expresión del presidente José Mujica que afirmaba "entre criollos nos entendemos" (se topeó el mercado para Directv, se impidió el acceso del grupo mexicano Slim y se redujo las bandas de frecuencias para el grupo argentino Clarín).

Por otra parte, en los gobiernos del Frente Amplio la televisión estatal no tuvo un mayor desarrollo como para impulsar el cambio de modelo televisivo (americano versus europeo) y por lo tanto los grupos mediáticos privados conservan su hegemonía de audiencias, facturación, etc.

Pese a que los gobiernos de turno, en los hechos, no hicieron perder ninguna de las empresas que ostentaban los grupos, el oligopolio exhibe su fragilidad por los desafíos que provienen de los desarrollos en las TIC y eso explica su insistencia por ampliar sus servicios al negocio de las transmisiones de datos. Como ya se expresó, en las reformas planteadas por el gobierno entrante se estaría habilitando esa posibilidad.

\section{5.- ¿CRónica de Una MUerte anUnCiada de las democracias o los muertos que vos MATÁIS GOZAN DE BUENA SALUD?}

Desde sus orígenes hasta nuestros días, la democracia liberal ha sido objeto de críticas sobre el verdadero alcance de la distribución del poder que prometía. Más allá de los fundamentos, la empiria viene señalando un debilitamiento en su legitimidad popular.

Estos datos se ponen a prueba a la luz de la emergencia sanitaria que nos afecta. Según los analistas de las más diversas perspectivas y disciplinas, coinciden en que el advenimiento de la pandemia y las cuarentenas impuestas a lo largo del planeta está provocando una crisis económicas sin precedentes y sus impactos en la conflictividad social e inestabilidad institucional prometen colocar a las democracias contra la pared. De hecho, se levantan implacables voces que ya auguran el fin del régimen y el capitalismo asociado, basados en referentes que informaban de una crónica de una muerte anunciada, como algunos movimientos intelectuales 
y políticos antisistémicos, y la evidencia de grandes sectores de la población mundial en condiciones marginales de los beneficios asignados al modelo capitalista.

En principio, sabemos que la llegada de las cuarentenas obligatorias o voluntarias han generado que los gobiernos dispongan de mayores recursos de poder y promuevan un despliegue mediático importante, fundamentalmente a través de los medios de masas tradicionales.

Desde la perspectiva deliberativa, en cambio, la democracia es el único régimen que garantiza un mínimo de libertades, protección de los derechos humanos y a los individuos para elegir los caminos que considere idóneos para su bienestar. Por lo tanto, proyectan un refortalecimiento democrático a partir de una expansión en las instituciones políticas y en la sociedad civil de la deliberación pública con el fin de mejorar la comunicación social en las decisiones colectivas a partir de razones e intercambios argumentativos. Desde la democracia deliberativa se vienen explorando diseños institucionales que permitan su proyección empírica. Pero más allá de los matices de esta corriente de pensamiento la deliberación, además de la voluntad de los gobiernos y de los políticos en general, hoy enfrenta un desafío mayor en el campo de las comunicaciones dado que sus nuevos desarrollos han fragmentado radicalmente las audiencias y por lo tanto, los argumentos comienzan a no ser dialógicos y la trazabilidad de la deliberación se vuelve confusa y parcial.

Hemos repasado algunos supuestos que explican este fenómeno, pero no han permitido aún resolverlos y de hecho, mucha de la literatura especializada no logra establecer alternativas de consenso.

De hecho, Uruguay pese a la cultura política que se le atribuye, no está exento de enfrentar duros desafíos democráticos como los que ya sufre el resto de la región. Investigaciones recientes han verificado que nuestros partidos políticos han recorrido largos períodos con amplios ambientes deliberativos que explican, en parte, la vigencia que aún conservan, la fortaleza institucional del estado que ellos han impulsado y la expansión de una cultura política en el conjunto de la sociedad, como avalan diversos estudios comparados.

El sistema mediático con el que Uruguay contó para sostener su perfil democrático no ha sufrido hasta el momento cambios sustanciales, más allá de algunos avances en materia regulatoria impulsada por los gobiernos del Frente Amplio y la expansión de la internet con algunas de sus aplicaciones que han potenciado el uso y acceso a las denominadas redes sociales. Para el caso uruguayo, podemos afirmar que los medios de masas tradicionales conservan su centralidad y permanecen autónomos de las organizaciones partidarias y sus intereses, aunque con negociaciones puntuales cuando de regulaciones y rediseño de mercado se trata. Fue así en cada uno de los hitos regulatorios y sobremanera ante cambios tecnológicos en materia de servicios de comunicación audiovisual.

En lo estrictamente ideológico no es una autonomía absoluta, en la medida que los grupos empresarios que los dirigen no ocultan algún tipo de sensibilidad ideológica que los operadores políticos suelen marcar y de hecho, en varios hitos descritos por investigaciones académicas (Beltramelli, 2018) dan cuenta de la utilización de la capacidad de fijar agenda que disponen para obtener beneficios corporativos frente a los gobiernos de turno en términos de licencias, asignaciones de frecuencias, autorizaciones de servicios y garantías o protecciones contra competencias emergentes.

Como efecto directo de la pandemia, en esta primera etapa es posible observar una revitalización de los medios tradicionales como fuente principal de información, pese a que aún no hay mediciones precisas que verifiquen algunos indicios, como el rating o la expansión de los espacios sobre debates de asuntos públicos en radios y televisión.

Sin embargo, para una segunda etapa y como consecuencia directa de las cuarentenas se prevé una crisis económica de magnitudes históricas. Ello permite inferir que los escenarios democráticos enfrentan nubarrones y desafíos muy importantes que pueden provocar una pérdida creciente de su calidad, cuando no, su caída como consecuencia de fenómenos como explosiones sociales y se exponen a su sustitución por sistemas autoritarios o semiautoritarios.

Uruguay no va a permanecer ajeno a esta realidad y sufrirá una pérdida económica sustantiva (se anuncia por parte de las nuevas autoridades una caída en el entorno al 3\% del PBI), aunque en primera instancia 
cuenta para sostener su democracia con instituciones sólidas como los partidos políticos, fuertes instituciones de gobierno y un sistema mediático plural ${ }^{9}$, aunque con escaso desarrollo de un sistema de medios públicos, sobre todo en lo que refiere a las audiencias televisivas.

Estos elementos permiten esperar que la crisis social no necesariamente derive en una crisis política aguda como para comprometer el sistema democrático sustantivamente.

En trayectos de más largo plazo, es menos predecible conocer los impactos que las reformas del nuevo gobierno puedan generar en los modelos de negocios y en su calidad democrática. La consolidación de infraestructuras instaladas por las administraciones anteriores han permitido una rápida adaptación de amplios sectores de la sociedad a los usos sobre internet para el teletrabajo y la educación a distancia, pero aún no es posible conocer sus permanencias y los efectos en el sistema económico con demasiado detalle. Es posible que se mantengan algunos recursos utilizados en este período extraordinario luego de la crisis y que los desarrollos para el e-governance se expandan, así como los beneficios (también efectos negativos en el factor trabajo) que estas tecnologías pueden aportar a algunos sectores específicos de la economía.

Mientras, en el sistema mediático tradicional parece haberse fortalecido en la primera etapa de la pandemia, pero cuando se instale fuertemente la crisis económica y las tensiones sociales comiencen a expresarse públicamente su utilización por parte de los diferentes actores del sistema va a ser crucial para establecer su proyección y poder observar si será capaz de conservar su hegemonía como fuente de información ciudadana.

Pero en materia de canales comunicacionales que favorezcan la proyección de ambientes de intercambios racionales con efectos en la esfera pública continúan siendo un interrogante sin respuesta, no solo para la academia, sino para el futuro de la sociedad uruguaya en el marco de su matriz cultural.

\section{ReFERENCIAS}

Alonso Bentos, E. (2019). Democracia deliberativa y partidos politicos: una aproximación histórica al caso uruguayo (Tesis de doctorado). Recuperado de https://hdl.handle.net/20.500.12008/23277

Beltramelli, F. (2018). Políticas públicas y convergencia infocomunicacional en Uruguay: regulaciones e institucionalidad en los gobiernos del Frente Amplio en Uruguay (2005-2014). La Plata: Universidad Nacional de La Plata.

Beltramelli, F., Alonso Bentos, E. y otros (2017). La ciudadanía ante la convergencia tecnológica y los medios de comunicación II. Montevideo, Uruguay: Ediciones Universitarias.

Biale, E. y Ottonelli, V. (2018). Intra-party Deliberation and Reflexive Control within a Deliberative System. Political Theory, 47(4), 500-526. https://doi.org/10.1177\%2F0090591718806829

Budge, I. (1993). Direct democracy: Setting appropriate terms of debate. En D. Held (Ed.), Prospects for Democracy. Cambridge: Polity.

Lanza, E. y Buquet, G. (2011). La televisión privada comercial en Uruguay. Caracterización de la concentración de la propiedad, las audiencias y la facturación. Montevideo, Uruguay: Friedrich-Ebert-Stiftung.

Califano, B. (2015). Perspectivas conceptuales para el análisis del Estado y las políticas de comunicación. Austral Comunicación, 4(2), 283-318. https://doi.org/10.26422/aucom.2015.0402.cal

Castells, M. (2000). La era de la información: economia, sociedad y cultura. La sociedad red. Vol. 1. Madrid, España: Alianza.

Chadwick, A. (2009). Web 2.0: New Challenges for the Study of E-Democracy in an Era oflnformational Exuberance. A Journal of Law and Policy for the Information Society, 5(1), 9-41.

Chambers, S. (2009). Rhetoric and the Public Sphere: Has Deliberative Democracy Abandoned Mass Democracy? Political Theory, 37(3), 323-350.

Dahl, R. (2009). La poliarquia. Participación y oposición. Madrid, España: Tecnos.

Dahlberg, L. F. (2000). The Internet and the public sphere: a critical analysis of the posibility of online discourse enhancing deliberative democracy (Tesis de doctorado). Nueva Zelanda, Massey University. 
Dryzek, J. (2009). Democratization as Deliberative capacity building. Comparative Political Studies, 42(11), 1379-1402. https://doi.org/10.1177\%2F0010414009332129

Fishkin, J. (1991). Democracia y deliberación. Nuevas perspectivas para la reforma democrática. Barcelona, España: Ariel.

Haberer, M. y Peña-López, I. (julio de 2016). Structural Conditions for Citizen Deliberation: A Conceptual Scheme for the Assessment of "New" Parties. En Building a European Digital Space. Actas del $12^{\circ}$ Congreso Internacional Internet, Derecho y Política (pp. 479-498). Barcelona, España: Universitat Oberta de Catalunya/ Huygens Editorial.

Habermas, J. (1998). Teoría de la acción comunicativa. Racionalidad de la acción y racionalización social. Madrid, España: Taurus.

Habermas, J. (1999). La inclusión del otro. Estudios de teoría política. Barcelona, España: Paidós.

Harto, F. (2006). Tipologies i models de democràcia electrònica. Revista de Internet, Derecho y Política, (2). http:// doi.org/10.7238/idp.v0i2.385

Katz, R. y Mair, P. (1995). Changing models of party organization and party democracy: the emergence of the cartel party. Party Politics, 1(1), 5-28.

London, S. (1995). Teledemocracy vs. Deliberative Democracy: A Comparative Look at Two Models of Public Talk. Journal of Interpersonal Computing and Technology, 3(2), 33-55.

Mazurca, E. C. (2005). Democracia deliberativa y teledemocracia: ¿Realidades enfrentadas o dos caras de un mismo discurso? En Actas del VII Congreso Español de Ciencia Política y de la Administración. Recuperado de https:// bit.ly/31IBHya

Negroponte, N. (1995). Ser digital. Ciudad Autónoma de Buenos Aires, Argentina: Atlántida.

Nielsen, J. (1993). Usability Engineering. Boston: Academic Press.

Sartori, G. (1999). Homovidens. La sociedad teledirigida. Ciudad Autónoma de Buenos Aires, Argentina: Taurus.

Schuliaquer, I. (2016). ¿Quién domina la escena? La relación entre los gobiernos del Frente Amplio y las empresas mediáticas en Uruguay (2005-2015). En E. Mitchelstein y P. J. Boczkowski (Comps.), Titulares, hashtags y videojuegos. La comunicación en la era digital (pp. 167-182). Ciudad Autónoma de Buenos Aires, Argentina: Manantial.

Subirats, J. (2011). Otra sociedad ¿Otra politica? De «no nos representan» a la democracia de lo común. Barcelona, España: Icaria Ediciones.

Subirats, J. (2015). El contexto de crisis y el cambio de época. En Centro Reina Sofía sobre Adolescencia y Juventud, Ya nada será lo mismo. Los efectos del cambio tecnológico en la política, los partidos y el activismo juvenil. Madrid, España: Fundación de Ayuda contra la Drogadicción.

Sunstein, C. (2001). Republic.com. Princeton, Estados Unidos: Princeton University Press.

Teorell, J. (1999). A Deliberative defense of intra-party democracy. Party Politics, 5(3), 363-382. https://doi.org/10 $.1177 \% 2 \mathrm{~F} 1354068899005003006$

Wessler, H. (2008). Investigating Deliberativeness Comparatively. Political Communication, 25, 1-22.

Wolkenstein, F. (2016). A Deliberative Model of Intra-Party Democracy. The Journal of Political Philosophy, 24(3), 297-320. https://doi.org/10.1111/jopp.12064

Young, I. M. (2000). La democracia y “el otro": más allá de la democracia deliberativa. (Trad. Lelia Mooney Sirotinsky). Revista Juridica de la Universidad de Palermo, 5(1). Recuperado de http://palermo.edu/derecho/publicaciones /pdfs/revista_juridica/n5N1-2000/051Juridica03.pdf

\section{Notas}

1 La teoría deliberativa se nutre de las aportaciones de autores de referencia como Manin (1987), Cohen (1989a), Fishkin (1991), Rawls (1996), Habermas (1994; 1998), Benhabib (1996), Bohman (1996), Gutmann y Thompson (1996), Elster (1998), Macedo (1999). En general, los autores que se reconocen dentro de las corrientes normativas de la democracia deliberativa varían en los diferentes trabajos pero algunos, como los mencionados se repiten en la mayoría 
2 Expresión extraída de la conferencia de prensa del presidente del Uruguay Luis Lacalle Pou, para informar sobre la conformación de un equipo de especialistas en proyectar escenarios post crisis sanitaria.

3 Todas estas instituciones miden regularmente a lo largo del tiempo a través de diversos indicadores la calidad de las democracias regionales o mundiales y sus informes o en algunos casos también sus bases de datos están disponibles on line.

4 Fishkin, James, 1997. Democracia y deliberación. Nuevas perspectivas para la reforma democrática. Editorial Ariel. Barcelona.

5 Sigla de Administración Nacional de Telecomunicaciones

6 https://www.impo.com.uy/bases/leyes/19307-2014

7 https://parlamento.gub.uy/documentosyleyes/documentos/repartidos. Repartido 43

8 En su artículo 48 el proyecto de la nueva Ley de Medios, establece la siguiente formulación: "Los titulares de licencias de servicios de difusión de contenido audiovisual de televisión para abonados que operan mediante cable, tendrán derecho a solicitar licencias para prestar simultáneamente servicios de banda ancha y acceso a Internet mediante el empleo de sus redes propias o de recursos que contraten con terceros. Todo lo cual será objeto de reglamentación. Los operadores públicos de telecomunicaciones que sean titulares de infraestructura, así como de redes de telecomunicaciones, prestarán servicios mayoristas sobre su infraestructura y redes a aquellos prestadores de servicios de televisión por abonados que operan mediante cable en el acceso o uso, para promover la inversión eficiente en el despliegue de redes”. El proyecto se encuentra a estudio del Legislativo desde mayo de 2020.

9 En el informe anual de Periodistas sin fronteras, Uruguay conserva el primer lugar de América del Sur en libertad de prensa. 